\title{
Frozen section diagnosis for axillary sentinel lymph nodes: the first six years
}

\author{
Mark L Mitchell \\ Department of Pathology, Christiana Care Health Services, Newark, DE, USA
}

\begin{abstract}
Intraoperative frozen section of axillary lymph nodes for the detection of metastatic breast cancer has been controversial because of the labor-intensive techniques necessary to obtain a highly sensitive test, and because of the uncertain significance of frequently detected submicrometastatic carcinoma. In total, 874 consecutive axillary sentinel lymph node cases examined by intraoperative frozen section over a 6-year period were reviewed retrospectively. Frozen section had a sensitivity of $60 \%$ and was $100 \%$ specific, but when cases with submicrometastatic tumor cells were considered negative, the sensitivity rose to $83 \%$. Rare cases were called 'atypical' on frozen section; almost all of these cases were negative for carcinoma on permanent sections.

Modern Pathology (2005) 18, 58-61, advance online publication, 10 September 2004; doi:10.1038/modpathol.3800279
\end{abstract}

Keywords: breast neoplasms; sentinel lymph node biopsy; frozen sections

Studies in the 1990s showed the value of the sentinel lymph node procedure for staging carcinoma of the breast. ${ }^{1-3}$ The technique identifies a sentinel lymph node or nodes in the axilla of a patient with breast carcinoma using a vital dye or radionuclide. If the sentinel lymph node is negative for malignancy, the patient can forego regular axillary lymph node dissection, since the probability that the additional dissected lymph nodes will contain metastatic tumor when the sentinel node is negative is about $5 \% .^{4,5}$ Examination of the sentinel lymph nodes has been done by frozen section at many institutions. Although of lower quality than permanent sections, frozen sections permit the patient to have evaluation of the sentinel node done during their operation and avoid a second procedure. Intraoperative frozen sections have been controversial because of the labor-intensive techniques necessary to obtain a highly sensitive test, and because of the uncertain significance of frequently detected submicrometastatic carcinoma.

This is a retrospective observational study of the use of frozen section for the examination of sentinel lymph nodes in 874 breast carcinoma patients from 1998 to 2004 at a large private nonprofit teaching medical center. The accuracy of the technique and

Correspondence: Dr ML Mitchell, MD, Department of Pathology, Christiana Care Health Services, Newark, DE 19718-6001, USA. E-mail: mmitchell@christianacare.org

Received 7 June 2004; revised and accepted 26 July 2004; published online 10 september 2004 the problems encountered with its use are discussed.

\section{Materials and methods}

Between April 1998 and 2004, sentinel lymph nodes were sent to the laboratory from 908 cases. Sentinel lymph nodes were identified using $450 \mu \mathrm{Ci}$ of filtered technetium sulfur colloid and $3-5 \mathrm{ml}$ of the vital dye isosulfan blue in the operating room. ${ }^{6}$ The lymph nodes were sent for frozen section diagnosis in 874 cases. Patients ranged in age from 32 to 78 years with a median age of 56 years. Lymph nodes were cut at approximately $2 \mathrm{~mm}$ intervals. The pieces were frozen and two or three hematoxylinand eosin-(H\&E) stained $6 \mu \mathrm{m}$ thick sections were made at each of two levels from each piece. Permanent sections from these blocks were stained with hematoxylin and eosin and examined at three additional levels. An additional level from the paraffin block was stained immunohistochemically with a pankeratin antibody (AE1-AE3, Ventana Corp., Tucson, AZ, USA). The routine use of the anti-keratin was discontinued in 2001, but some pathologists continued to use anti-keratin in certain cases (eg lobular differentiation in the primary tumor, see discussion). Nonsentinel lymph nodes were also cut at $2 \mathrm{~mm}$ intervals and submitted entirely, but H\&E-stained sections were produced from only one level from each block. Confidence intervals for sensitivity and specificity were calculated using the Wilson score method. ${ }^{7}$ 


\section{Results}

The number of sentinel lymph nodes ranged from 1 to 12 , with a mean of 2.4 lymph nodes per case. Nonsentinel lymph nodes ranged from 0 to 34 in number with a mean of 8.5 lymph nodes. The cases were submitted by 32 different surgeons, but $62 \%$ of the submitted cases came from only three of the surgeons. Cases were examined by one of 11 different pathologists.

Table 1 shows the number of positive and negative frozen section results and the corresponding result on permanent section. In all, 16 of the cases were called atypical on frozen section. Overall, 68 of the cases that were negative on frozen section, had positive permanent sections that contained only small numbers of anti-keratin-positive cells (54 cases) or H\&E-positive cells (14 cases). These cases were reviewed and all had tumor measuring less than $0.2 \mathrm{~mm}$ and most would be staged as 'pNo(i+) sn' in the TNM system. Since there is some evidence that these cases do not represent clinically important metastases, Table 2 shows the data with these cases classified as negative.

Table 3 shows the sensitivity, specificity, overall accuracy (efficiency, concordance rate), false reassurance rate and false alarm rate for the data in Tables 1 and 2 . The 95\% confidence intervals for sensitivity were $0.54-0.66$ for Table 1 data and $0.77-$ 0.87 for Table 2 data. The overall accuracy is the proportion of all cases that were correctly called either positive or negative on frozen section. The false reassurance rate is the proportion of cases called negative on frozen section that was positive

Table 1 Sentinel lymph node frozen section diagnoses vs permanent section diagnoses

\begin{tabular}{lrcc}
\hline & $\begin{array}{c}\text { Positive } \\
\text { SLN }\end{array}$ & $\begin{array}{c}\text { Negative } \\
\text { SLN }\end{array}$ & Row totals \\
\hline Positive frozen section & 153 & 0 & 153 \\
Atypical frozen section & 6 & 10 & 16 \\
Negative frozen section & 94 & 611 & 705 \\
Column totals & 253 & 621 & 874 \\
\hline
\end{tabular}

Submicrometastatic carcinoma $(<0.2 \mathrm{~mm})$ called positive.

Table 2 Sentinel lymph node frozen section diagnoses vs permanent section diagnoses

\begin{tabular}{lrrr}
\hline & $\begin{array}{c}\text { Positive } \\
\text { SLN }\end{array}$ & $\begin{array}{c}\text { Negative } \\
\text { SLN }\end{array}$ & Row totals \\
\hline Positive frozen section & 153 & 0 & 153 \\
Atypical frozen section & 3 & 13 & 16 \\
Negative frozen section & 29 & 676 & 705 \\
Column totals & 185 & 689 & 874 \\
\hline
\end{tabular}

Submicrometastatic carcinoma $(<0.2 \mathrm{~mm})$ called negative.
Table 3 Sensitivity and specificity of frozen section diagnosis of sentinel lymph nodes

\begin{tabular}{lcc}
\hline & Table 1 data & Table 2 data \\
\hline Sensitivity & 0.6 & 0.83 \\
Specificity & 1 & 1 \\
Overall accuracy & 0.89 & 0.96 \\
False reassurance rate & 0.14 & 0.04 \\
False alarm rate & 0.63 & 0.81 \\
\hline
\end{tabular}

on permanent section, that is, cases in which the surgeon was falsely reassured. The false alarm rate was created because of the unusual finding that some of the frozen sections had been called atypical. It is the proportion of frozen sections called atypical that were negative on permanent section, that is, cases in which the surgeon was given a false alarm.

The 16 cases called 'atypical' on frozen section were reviewed by the author. Two cases were thought to have diagnostic carcinoma and had been given an under diagnosis originally, and two cases had probable carcinoma that was difficult to diagnose conclusively. Of the two cases with probable carcinoma on review, one had a few antikeratin-positive cell clusters in the permanent sections and one had no evidence of tumor in permanent sections by H\&E or anti-keratin stains. The other 12 cases had various appearances including some thought to represent histiocytes, highendothelium venules, and unusual sections of germinal centers.

\section{Discussion}

Since the popularization of the sentinel lymph node procedure for breast carcinoma, intraoperative examination of axillary sentinel lymph nodes by frozen section has been controversial. This has been because there is a strong desire to perform the sentinel lymph node biopsy and the subsequent axillary node dissection during a single procedure, but the reported sensitivity of frozen section for the detection of carcinoma in the sentinel node has ranged as low as $54 \% .{ }^{8}$ In a study by Veronesi et $a l^{2}$ in which sentinel lymph nodes were bisected and only one-half of the lymph node was frozen, frozen section diagnosis had a sensitivity of $64 \%$ with an overall accuracy of $83 \%$.

Attempts to improve the sensitivity of intraoperative examination have included step sectioning the lymph node, using anti-keratin immunohistochemistry at the time of frozen section, and using cytological preparations from the sentinel lymph node. $^{4,9,10}$ Some of these techniques increase the laboratory workload at the time of frozen section quite dramatically, but sensitivities (in reports with more than 100 patients) have been as high as $93 \% .{ }^{4}$ Recently, Krogerus compared two frozen section 
techniques. ${ }^{11}$ Lymph nodes were either bisected and cut twice at each of five levels or they were cut at a thickness of $1-1.5 \mathrm{~mm}$ with a razor blade and two sections and touch preparations were made from each piece. The authors claimed that the second method was less labor intensive and found more metastases, but the sensitivity for detection of carcinoma was actually higher using the first technique, since there were no false-negative cases.

In our laboratory a compromise technique was developed in which lymph nodes for frozen section were cut at $2 \mathrm{~mm}$ thickness and two sections were cut at each of two or three levels and stained with hematoxylin and eosin. Our data show that frozen sections performed by 11 different pathologists during a 6-year period were highly specific, but only moderately sensitive for the detection of metastatic carcinoma in axillary sentinel lymph nodes. There was no case with a falsely positive frozen section, but in 94 cases, the frozen section was called negative and the subsequent permanent sections contained carcinoma cells $(13.3 \%$ of negative frozen section cases). Sentinel lymph nodes were positive for metastatic carcinoma of any size in $29 \%$ of cases.

The controversy over the use of frozen sections on sentinel lymph nodes has been further complicated by the discovery of increasing numbers of lymph nodes with very small numbers of tumor cells. Prior to the use of the sentinel lymph node technique, routine histologic examination of axillary lymph nodes examined only a small portion of the node. It has been known for many years that routine lymph node examination understages a significant proportion of breast cancer patients. ${ }^{12}$ It was thought that the sentinel lymph node technique would eliminate this problem by permitting the laboratory to concentrate its resources on the careful examination of a small number of lymph nodes. Unfortunately, many of the positive sentinel lymph nodes detected with step sections and immunohistochemistry have had very small numbers of tumor cells, and the clinical significance of micrometastatic carcinoma $(0.2-2.0 \mathrm{~mm})$ and submicrometastatic carcinoma $(<0.2 \mathrm{~mm})$ is uncertain. Studies on the clinical significance of these cells have had mixed results, ${ }^{13-17}$ and the results of long-term follow-up are not yet available. A recent study by Moore et al ${ }^{18}$ found evidence that sentinel lymph node tumor cells detected by immunohistochemistry only, might be due to so-called benign transport, and not represent true metastases. Our department confronted the problem of the increasingly frequent finding of individual anti-keratin-positive tumor cells in sentinel lymph nodes in 2001, when some of the pathologists and staff oncologists were concerned that these cells might not represent clinically significant metastases. These patients, staged as $\mathrm{No}(\mathrm{i}+)$ sn in the TNM system, were not being treated as having positive axillary lymph nodes in the community, but there was considerable patient anxiety from the diagnosis. Routine use of anti-keratin immunohistochemistry on sentinel lymph nodes was discontinued at that time as recommended by at least some authorities. ${ }^{19}$ Antikeratin continued to be used in cases with lobular differentiation in the breast primary tumor, and in cases with other atypical features.

When cases with submicrometastatic tumor were eliminated from the false-negative cases in our data, frozen section sensitivity rose from 0.60 to 0.83 and the overall accuracy rose from 0.89 to 0.96 . This is expected, since frozen sections in general have had an accuracy of $0.89-0.99,{ }^{20}$ and the key difference between sentinel lymph nodes and a more typical lymph node frozen section is that the pathologist is trying harder to detect a smaller amount of tumor. These values also indicate that intraoperative frozen section diagnosis is a useful technique, especially if patients with only submicrometastatic carcinoma are not considered to have positive lymph nodes. By using frozen sections, 153 patients in our study had axillary lymph node dissection at the same time as their excisional biopsy and 32 patients had to have a second procedure because of a falsely negative frozen section. But if frozen section had not been used, all 185 patients would have had a second procedure.

An interesting phenomenon occurred at the time that routine immunohistochemical staining was stopped. The number of cases called 'atypical' on frozen section began to increase. This occurred despite the well-known dictum for frozen section diagnosis, 'In cancer there are only three possible diagnoses: positive for cancer, negative for cancer, or no diagnosis made', ${ }^{21}$ and was probably due to the perceived pressure to find carcinoma cells in the sentinel lymph node. Of the 16 cases called atypical on frozen section, only three had carcinoma on permanent sections. This gives a 'false alarm' rate of 0.81 , indicating that the atypical diagnosis on sentinel lymph node frozen sections was not helpful.

\section{References}

1 Giuliano AE, Kirgan DM, Guenther JM, et al. Lymphatic mapping and sentinel lymphadenectomy for breast cancer. Ann Surg 1994;220:391-398; discussion 398-401.

2 Veronesi U, Paganelli G, Galimberti V, et al. Sentinelnode biopsy to avoid axillary dissection in breast cancer with clinically negative lymph-nodes. Lancet 1997;349:1864-1867.

3 Albertini JJ, Lyman GH, Cox C, et al. Lymphatic mapping and sentinel node biopsy in the patient with breast cancer. JAMA 1996;276:1818-1822.

4 Viale G, Bosari S, Mazzarol G, et al. Intraoperative examination of axillary sentinel lymph nodes in breast carcinoma patients. Cancer 1999;85:2433-2438.

5 Veronesi U, Paganelli G, Viale G, et al. Sentinel lymph node biopsy and axillary dissection in breast cancer: 
results in a large series. J Natl Cancer Inst 1999;91: 368-373.

6 Dickson-Witmer D, Witmer DR, Hornung J. SLN biopsy in breast cancer: Christiana care experience. May 1998-February 1999. Del Med J 1999;71:391-397.

7 Newcombe RG. Interval estimation for the difference between independent proportions: comparison of eleven methods. Stat Med 1998;17:873-890.

8 Kane III JM, Edge SB, Winston JS, et al. Intraoperative pathologic evaluation of a breast cancer sentinel lymph node biopsy as a determinant for synchronous axillary lymph node dissection. Ann Surg Oncol 2001;8: 361-367.

9 Motomura K, Inaji H, Komoike Y, et al. Intraoperative sentinel lymph node examination by imprint cytology and frozen sectioning during breast surgery. Br J Surg 2000;87:597-601.

10 Noguchi M. Sentinel lymph node biopsy and breast cancer. Br J Surg 2002;89:21-34.

11 Krogerus LA, Leidenius $\mathrm{MH}$, Toivonen TS, et al. Towards reasonable workload in diagnosis of sentinel lymph nodes: comparison of two frozen section methods. Histopathology 2004;44:29-34.

12 Saphir O, Amromin GD. Obscure axillary lymph-node metastasis in carcinoma of the breast. Cancer 1948; 1:238-241.

13 Noguchi M. Therapeutic relevance of breast cancer micrometastases in sentinel lymph nodes. Br J Surg 2002;89:1505-1515.
14 Allred DC, Elledge RM. Caution concerning micrometastatic breast carcinoma in sentinel lymph nodes. Cancer 1999;86:905-907.

15 Dowlatshahi K, Fan M, Bloom KJ, et al. Occult metastases in the sentinel lymph nodes of patients with early stage breast carcinoma: a preliminary study. Cancer 1999;86:990-996.

16 Viale G, Maiorano E, Mazzarol G, et al. Histologic detection and clinical implications of micrometastases in axillary sentinel lymph nodes for patients with breast carcinoma. Cancer 2001;92:1378-1384.

17 Giuliano AE, Kelemen PR. Sophisticated techniques detect obscure lymph node metastases in carcinoma of the breast. Cancer 1998;83:391-393.

18 Moore KH, Thaler HT, Tan LK, et al. Immunohistochemically detected tumor cells in the sentinel lymph nodes of patients with breast carcinoma: biologic metastasis or procedural artifact? Cancer 2004; 100:929-934.

19 Schwartz GF, Giuliano AE, Veronesi U. Proceedings of the consensus conference on the role of sentinel lymph node biopsy in carcinoma of the breast, April 19-22, 2001, Philadelphia, Pennsylvania. Cancer 2002;94: 2542-2551.

20 Oneson RH, Minke JA, Silverberg SG. Intraoperative pathologic consultation. An audit of 1,000 recent consecutive cases. Am J Surg Pathol 1989;13:237-243.

21 Ackerman LV. Surgical Pathology. 3rd edn. The C.V. Mosby Company: St Louis, 1964, p 24. 